\title{
Engaging Learners in Dialogue Interactivity Development for Mobile Robots ${ }^{\star}$
}

\author{
Paul Baxter ${ }^{10000-0002-7299-9234]}$, Francesco Del \\ Duchetto $^{1[0000-0002-3627-317 X]}$, and Marc Hanheide ${ }^{1[0000-0001-7728-1849]}$ \\ L-CAS, School of Computer Science, University of Lincoln, UK \\ \{pbaxter, fdelduchetto, mhanheide\}@lincoln.ac.uk \\ https://lcas.lincoln.ac.uk
}

\begin{abstract}
The use of robots in educational and STEM engagement activities is widespread. In this paper we describe a system developed for engaging learners with the design of dialogue-based interactivity for mobile robots. With an emphasis on a web-based solution that is grounded in both a real robot system and a real application domain - a museum guide robot - our intent is to enhance the benefits to both driving research through potential user-group engagement, and enhancing motivation by providing a real application context for the learners involved. The proposed system is designed to be highly scalable to both many simultaneous users and to users of different age groups, and specifically enables direct deployment of implemented systems onto both real and simulated robots. Our observations from preliminary events, involving both children and adults, support the view that the system is both usable and successful in supporting engagement with the dialogue interactivity problem presented to the participants, with indications that this engagement can persist over an extended period of time.
\end{abstract}

Keywords: Mobile Robots · DialogFlow · Museum Guide · Public Engagement · Dialogue Interactivity

\section{Introduction}

Robots have long formed a useful educational tool in part by making computation tangible and accessible [12], as physical instantiations of Papert's communication with computers [13]. We are concerned here with robots acting as educational platforms that the learners control, rather than the more recent endeavours to use social robots as learning companions, for which a different set of considerations apply, e.g. [1]. More specifically, we are interested in encouraging engagement with the high-level control of robots (rather than the low-level control of teleoperated movement for example) such as social interactivity, and the concepts involved in programming these into robots.

\footnotetext{
* The work described in this paper was supported by the EU H2020 ("ILIAD", contract 732737), EPSRC ("NCNR" Hub EP/R02572X/1), and the Johnson and Mukherjee Brothers Charitable Trust.
} 
The work we present in this paper is part of a wider research programme concerned with improving the long-term autonomy of mobile robots in humancentred environments, as an extension of prior and continuing research $[7,4]$. Of specific interest to the current effort is to increase the social interactivity of the robots concerned so as to reduce barriers to interaction and increase utility for non-technical users. One application domain of particular interest is the use of a mobile robot to interact with the general public in a museum, to help guide, provide information, and engage members of the public with the exhibits. The general research context for the present paper is that one potentially valuable source of information on interactions with the public can be obtained through the public's engagement with robot-centred STEM activities.

This work is conducted in collaboration with the Collection Museum ${ }^{1}$, which houses exhibits related to local archaeology and history. In an ongoing collaboration, the intention is for a robot to be permanently based in the museum, to act as a guide, and to provide further information on exhibits to interested visitors. In order to achieve this, the robot should engage members of the public who have no guaranteed prior experience with robots, and be able to respond to queries in an appropriate manner. While the robots are equipped with a touchscreen that may be used for interaction (section 3.3), the intention is to enable verbal interactions, thus necessitating dialogue management, since verbal interactivity is an important means of guiding users' expectations of competence [6].

In this paper, we bring together these strands of educational support through engagement with robotics, and research efforts to increase social interactivity of autonomous robots. By integrating these efforts, we seek to enhance the benefits to both - in terms of driving research through engagement with potential user groups (towards the manner of participatory design[14] for example), and enhancing motivation by providing a real application context for the learners involved.

We introduce a system that supports this integrative effort: it provides a tool to engage with the complexities of human-like dialogue, and links this to both real and simulated robots so that these issues can be directly evaluated. Being a web-based application, this does not require the installation of specialist software (beyond a standard web browser), and enables the simultaneous participation of large numbers of users. As a result, the learners not only explore issues related to social interactivity in our application domain, but also gain hands-on experience with the latest technology used for dialogue interactivity with computers more generally. We intend this paper to act as a resource for those who wish to use and evaluate (beyond our case studies) the system we present: to facilitate this, all code sources and exercises are provided.

\section{Background}

Robot programming in the educational contexts of interest here have typically focused on using robots to physically act out the implementation of algorithms (e.g.

\footnotetext{
${ }^{1}$ https://www.thecollectionmuseum.com/
} 
controlling movement). From the basic use of programming languages, tangible devices were introduced for such implementations [12]. More recently graphical programming languages ( vide accessible means of learning programming principles, despite some evidence of superior engagement with tangible devices [8]. The intent in these is to enable non-experts to engage with and learn the basic concepts and control, particularly where robots are concerned.

A range of methods have been employed in terms of programming a robot to achieve desired tasks. In addition to explicit and graphical programming, where a high level of platform familiarity and programming expertise is required, there have been increasing efforts to have the robots learn from demonstration, e.g. [2]. An approach such as this more explicitly acknowledges the role that non-robotics-experts can play in increasing the competence of robots. This acknowledgement continues with programming methods that abstract away from low-level algorithms and focus on behaviours, e.g. for social robots [5].

A number of robots have in the past been used in the domain of museums for guiding visitors. A notable example is the Minerva robot [15], which allowed the general public to control the robot through a web interface, enabling both influence of high-level strategy (choosing a visitor tour), or by explicitly choosing a predefined location in the museum for the robot to navigate to. While the robot was capable of some social interaction and was able to learn during interactions, this could not be explicitly influenced by the public controllers. More recent applications have, for example, focused on adding more human-like guiding behaviours to robot tour guides, with the intention of improving the experience for museum attendees [9]. Results indicate that human-like communication is used when interacting with robots [10].

The challenge thus remains improving the social interactivity of the robots that help the visitors. As noted above, we focus on language-based interactivity, and how we can engage naive users, whether they are children or adults, with the fundamental issues involved and potential solutions.

\section{$3 \quad$ System Setup}

In order to improve the dialogue handling capability of our museum guide robot, we have designed a system that is comprised of three main elements (figure 1). First is DialogFlow, a tool (now under the Google umbrella) to design, implement, and host conversational interfaces (whether these are text or speech based). Second is a robot system, in our case operating on the Robot Operating System (ROS), which can either be real or simulated, to which implemented DialogFlow behaviours are linked. Finally is a fulfilment server that link these two together. In the following subsections, we briefly describe each of these components. The source code is freely available for inspection and use ${ }^{4}$.

\footnotetext{
${ }^{2}$ https://scratch.mit.edu/

${ }^{3}$ https://developers.google.com/blockly/

${ }^{4}$ https://github.com/LCAS/robot-dialogflow/
} 


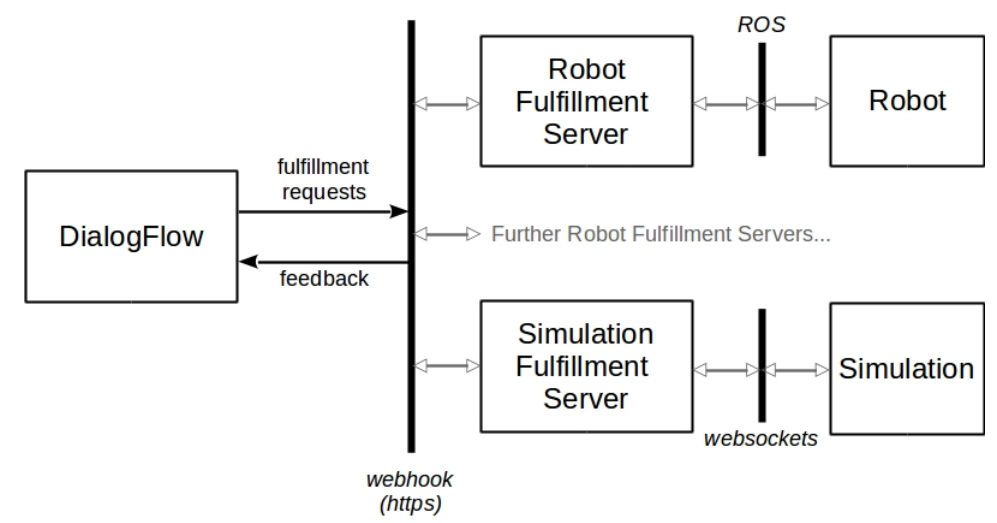

Fig. 1: System overview: the robot and the simulation can be swapped without behaviour reconfiguration by simply defining an alternative fulfillment server. The robot fulfillment server and robot communicate through ROS, and the simulation fulfillment server and the simulation through web sockets. The interaction between DialogFlow and the fulfillment server uses a webhook (https).

There are three main benefits to using a system such as this. Firstly, due to the availability of the simulated version of the robot and environment, the activities can be completed anywhere with a computer and an internet connection. This means it is versatile and applicable for the vast majority of engagement scenarios (from school visits to/from University, workshops at summer schools/camps, etc). Secondly, the online nature of the tools used means that the engagement from participants can continue beyond the formal activity time. This is an important facet of the current system, as it facilitates ongoing interaction and learning for the participants on their own terms. Finally, it enables robot behaviours developed in simulation, and potentially remotely, to be applied to a real robot in a real environment with minimal alteration. This is a useful feature in terms of applicability to the research context, but it can also be used to support motivation through the potential for the fast deployment of participant behaviours.

\subsection{DialogFlow}

Handling natural language is an important feature for a robot intended to interact with the public. While natural language processing encompasses a wide range of technical challenges [3], the aspect that we are concerned with particularly is dialogue management, which is concerned more specifically with linking the current state of the conversation with actions (which could include further dialogue) [11]. There are a wide range of approaches and tools available for doing this. For reasons of availability, usability, and scalability, we employ DialogFlow ${ }^{5}$,

\footnotetext{
${ }^{5}$ https://dialogflow.com/
} 


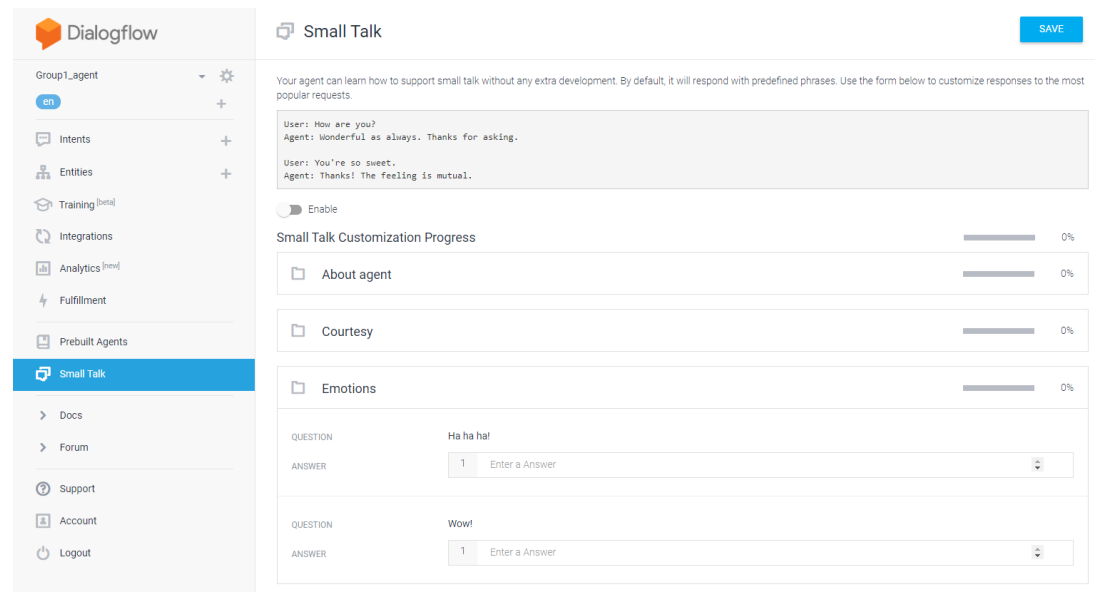

Fig. 2: A screenshot of the DialogFlow page showing the smalltalk definition area for one of the participant groups (section 4.1).

a Google-owned tool that facilitates the design of appropriate responses for conversational agents, and which is used in the Google Assistant ${ }^{6}$. In our system (figure 1), we use DialogFlow not only to generate appropriate verbal responses, but also to assess what robot actions are most appropriate (see section 3.2).

The essential components of a DialogFlow agent that users can define are Intents and Fulfillments. Intents define the relationship between the input (for example a spoke command to the robot) and the desired action. Creating Intents takes the form of defining a phrase (or set of phrases) that should be recognised, and then defining what should be done if a recognition occurs. Fulfillments allow the actions to be executed and are used to send commands to the robot (see section 3.2).

One basic example of interactivity that can be defined in DialogFlow is smalltalk (figure 2). In the context of our system, smalltalk does not result directly in robot action, but can be extended to give a greater degree of verbal interactivity. This is one aspect that we focus on in particular in our engagement with participants.

While at present a freely available resource, the use of DialogFlow does pose a number of practical challenges, particularly if the system is used by children. Primarily, a Google user account is required, which places restrictions on who can use the system, for example a minimum age restriction. There are however various circumstances in which children can make use of such services, particularly in educational contexts. This issue can also be circumvented by using an alternative dialogue planning system, or indeed a more basic hand-coded solution (in which all possible speech variations must be explicitly coded by hand), in place of DialogFlow. This is principle does not change the overall system schema, al-

\footnotetext{
${ }^{6}$ https://assistant.google.com/
} 


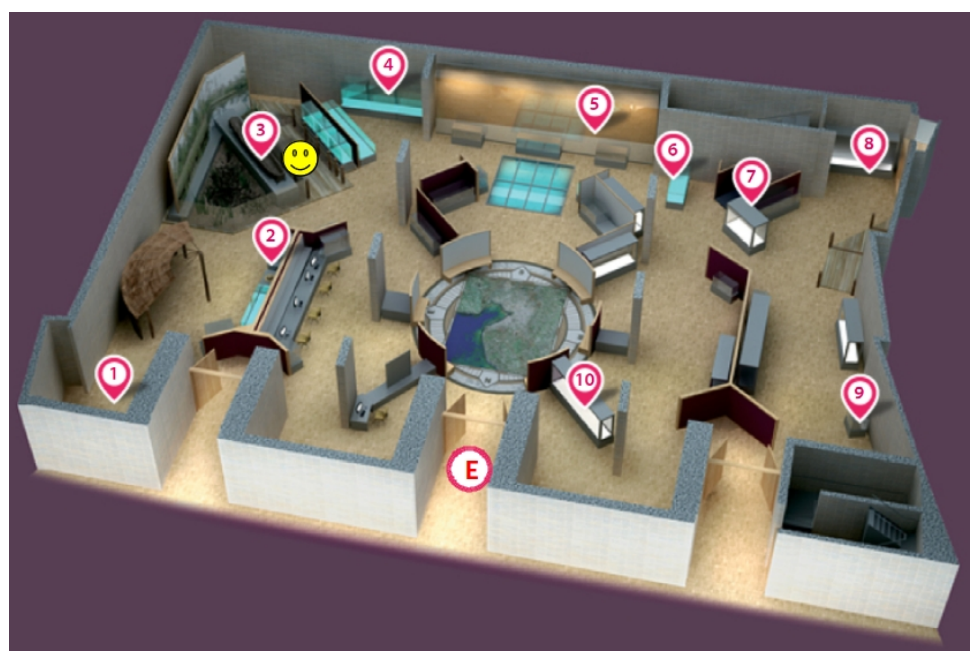

Fig. 3: A simulation of the museum in which the robot should be controlled, with multiple locations of interest shown that are targets for robot navigation. The robot is represented in this map with a smiley face (that can be displayed with eyes open or shut).

though it potentially reduces the inherent flexibility of the resulting system given that the background machine learning for interpretation of language input in the back-end of DialogFlow would no longer be used.

\section{$3.2 \quad$ Fulfilment Server}

The fulfillment server is an interface between the DialogFlow agent and the robot actions and events. A set of commands were predefined relating to robot movement around the museum space (e.g. go to one of 11 predefined locations, figure 3, comprised of 10 exhibits and the entrance), behaviours (e.g. open/close eyes), and the display of information (e.g. displaying information on the robot touchscreen, figure 4(a), or reporting the current location back to the DialogFlow agent). Importantly, due to the system setup (figure 1), these commands can be sent to either the real robot, or to the simulated robot (figure 3), with the difference simply being the definition of a webhook to access a web service hosted by the desired platform (figure 1). This allows the same DialogFlow agent to be easily repurposed.

\subsection{Robot}

The target robot platform for development in our application is based on the Scitos G5, extended with additional sensors and computing capacity. A mobile robot with low ground clearance, good stability, and standing at approximately 


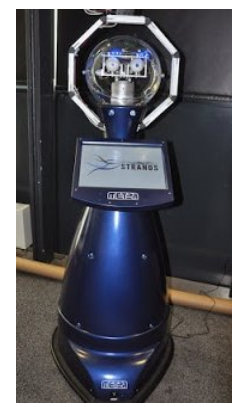

(a) A modified version of the Scitos G5 mobile robot.

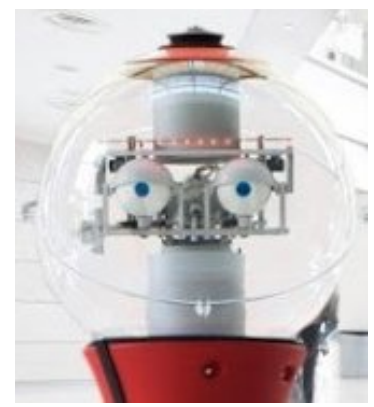

(b) Some facial expressivity, namely eye blinking and gaze direction.

Fig. 4: The robot used for the case studies presented.

$1.6 \mathrm{~m}$ tall, it is ideal for moving in indoor environments, and for interacting with people. It has a limited capacity for facial expressivity, extending to blinking eyes and manipulation of gaze direction (within a static head, see figure 4). The robot is controlled using the ROS middleware, which as a technical standard, facilitates integration with a range of specialist processing components (e.g. for navigation or visual processing) and interoperability with a range of other robot platforms.

Within the overall system however, the precise robot used is not important, provided that certain basic functionality is fulfilled, namely navigation and sufficient sensor capability for interaction with humans (e.g. microphone for speech recognition, and camera for person/face localisation). This arrangement, partially afforded by the use of ROS, means that a simulated version of the robot can be used in place of a real one (figure 3 ).

\section{Case Studies}

Using the system described above, we engaged learners in two events. Each of these were with different profiles of participants, in terms of both age and background, although the same system was used in both cases. In both cases, post-event questionnaires were given to the participants in order to gain insight into their experiences. Full details of these questionnaires and the responses are available $^{7}$.

\subsection{School Children}

Context: In a large scale STEM engagement event, we hosted nearly 20010 year old children from primary schools in the local area over the course of two

\footnotetext{
7 https://paul-baxter.github.io/pdf/supplementary/2018-EduRobotics-Supplementary. pdf
} 
days (just under 100 on each day). The aim was twofold. Firstly to engage the children with technology and applications beyond their typical school experiences through engagement with robotics and artificial intelligence. Secondly to have the children involved in the design of interactive robot behaviours that could be potentially deployed on the real robot (the children were made aware of this possibility).

Participants: A total of 188 children from 14 local primary schools took part in this event. All participating children were in U.K. year 5, and were thus nine or ten years old, with the majority ten (due to the time of the school year this activity took place - mean 9.606, s.d. 0.505). The intention was that the activities would be targeted at schools whose pupils were of a lower socio-economic status, thus providing the children with an opportunity not normally available to them. There was an even balance of boys (50.7\%) and girls (49.3\%). The children were divided into a total of 36 groups, with group members within the same school, and resulting group sizes from 5 to 8 members.

Process: The event took place over two days, where the second day was a repeat of the first, and each child only attended one of the two days. The event was situated in a large computer lab, typically used by undergraduates. Each group of children were given access to two desktop computers, with the instruction that one was to be used for the simulation and programming, and the other to be used for displaying the instructions/worksheets. There were seven stages of the activity covered over the course of the day, each with an associated worksheet, with a mix of paper-based and computer-based tasks ${ }^{8}$ :

1) Group organisation and team name (paper-based, team name was applied to their developed DialogFlow system)

2) Computer setup (intialising DialogFlow and the simulation)

3) Assessing variability in speech (paper-based, thinking about people can say the same thing in different ways)

4) Small talk (applying the speech variability ideas to small talk definition - see figure 2 for an example)

5) Starting to define robot behaviours (Phrases and Fulfillments)

6) Thinking about the strategic level of the desired robot behaviour

7) Turning planned behaviours into Intents

Throughout the day, there was a robot present in the room to contextualise the eventual task (see figure 4) - various behaviours for this robot were demonstrated during the lunch break. At the end of the event day, the children were asked to complete an online questionnaire (using one of the computers available to their group), this was voluntary, and with the only identifiable information requested the name of the school they attended (for the purpose of further analysis by school - this is not covered in the present work). The children (and their teachers) were encouraged to continue development of their solutions from their schools: both the worksheets and the simulation setup remained online to facilitate this.

\footnotetext{
8 The worksheets may be found at the following: https://sites.google.com/view/ uol-ai-robotics/materials
} 
Outcomes: Qualitatively, both teachers and children verbally reported good levels of engagement and enjoyment throughout the event. This is supported by the post-event questionnaire, with a response rate of $76.6 \%$ (144 responses in total). Overall, the children reported enjoying the event (1.45 mean, 5 -point scale where 1 is very positive), which is consistent with an overall positive opinion on robots (1.92 mean), and with a positive response to the idea of having a robot help them in various contexts, such as in a museum (1.63 mean), at school (1.69 mean), and at home (1.9 mean). The free-text responses provide some further insight, analysed in terms of keyword frequency analysis (please refer to the supplementary materials for all the data). The term "Robot/s" were identified as the aspect most enjoyed, even though the presence of real robots was only a small part of their experience during the event. This is consistent with them identifying both "robot/s" and "programming" as aspects they learned, with these keywords appearing more frequently than when asked what they liked most about the day. In terms of negative aspects of the experience, where present (the keyword with the highest use was "nothing"), these mostly related to challenges of working in a group.

Discussion: To a certain extent, it is relatively unsurprising that the children indicated that they had enjoyed the event, given that it involved a day away from school interacting with technologies they were previously unfamiliar with. It is interesting to note that the children identified "robot/s" and "programming" with greater frequency with respect to learning outcomes (122 and 61, respectively) than with respect to their enjoyment (81 and 43 , respectively). Although this observation will require further investigation with respect to real learning/skill gains, it is an initial indication that the children were aware of being engaged in a learning process.

\subsection{Undergraduate Students}

Context: As part of a summer school (University of Valladolid, Spain), a twohour activity was run for participants. The general topic was interactive robotics, with the aim of familiarising the participants with the associated methods and technologies. In this case, the focus was on expressive dialogue interactivity for (social) robots.

Participants: There were a total of 14 participants (and two professors), most of whom were undergraduate level. All were attendees at the summer school, and opted in to participate in the activity. According to a self-reported level of experience in the topic, there was only middling pre-existing familiarity declared (3.37 mean on a 5 point scale, 1 being high familiarity), consistent with the undergraduate level.

Process: The activity was essentially a compressed version of the day-long activity presented to children (section 4.1), though with the emphasis on individual work, rather than group work. Due to the location of the workshop, there was no physical robot present, and so only the simulation environment was used. However, the same principles applied as for the children's event. 
Outcomes: As with the children's event, self report feedback forms were administered to all participants (with full results shown in the appendix): eight participants returned these. A high level of satisfaction with the event (1.63 mean, 5-point scale where 1 is positive) was reported, and an agreement with both the format of the activity (1.63 mean) and the content of the activity (1.75 mean). Qualitatively, a good level of engagement with the delivery team was experienced, consistent with the feedback from responses.

Discussion: While involving participants at a higher level of education and experience, this case study demonstrates the applicability of our dialogue design for mobile robots system beyond children. The emphasis of such an engagement naturally changes, focusing more on implementation details than the foundational concepts, but the system itself remained identical to the previous case study, as indeed did the principles of the problem presented to them with the focus on dialogue interactivity for real robots.

\section{General Discussion}

In this paper, we have motivated and presented a system to engage learners with the design of interactive systems, in our case specifically to increase the social interactivity of museum guide robots. This system employs a freely available commercial interface for designing behaviours, but has been interfaced to a backend that facilitates the execution of behaviours seamlessly on both real and virtual robots. Two case studies were described: one large scale involving nearly 200 ten year old children, and one smaller in scale involving 14 undergraduate students. Despite the large disparity in participant groups, in both cases the same system was used (section 3). The metrics we employed to inspect engagement and enjoyment (self-report measures) demonstrated success for both. We are clear that these metrics can only be interpreted as indicators for engagement with the learning content of the respective activities - however, the data obtained is consistent with this perspective.

What we have not yet done as part of this research is make any assessment of learning outcomes, beyond the immediate self-reflection of the children. Since we were concerned with skill development and engagement, this would have necessitated both a longer-term perspective, and a clearer notion of definitive outcome for the respective participants. Since this work is ongoing, with a final engagement event for the first case study with children yet to take place (approximately six months after the initial activity days), one aspect that we intend to inspect is the relative complexity of the solutions (i.e. DialogFlow agent behaviours) developed by the teams of children. While this would not be a complete means of assessing learning outcomes, it would provide insight into continued engagement beyond the initial activity, and relative mastery of the tools available to them.

Our system and application to the two events have demonstrated consistency with the principles of previous applications of robotics to education. What we further emphasise here, through the tie-in to a real application domain, is the link 
to ongoing research efforts. This has the intention of being mutually beneficial, and adding a level of motivation/engagement for the learners as a result.

There are aspects of the system that could be extended to take further advantage of the features we identify as central to the approach proposed in this paper. Primary among these would be the provision of a higher-fidelity simulation environment that is similarly accessible through a web browser. This would facilitate both a greater immersive experience when using the simulator, but also a more realistic view of the defined robot behaviours, which in turn would allow a greater degree of detail in the designed interactive behaviours. Ensuring accessibility through a web browser, as we have currently achieved, would expand the set of the general public (including schools) able to engage with these activities, as no specialist software installations are required. If implemented in such a way as to maintain compatibility with a real robot platform (which our use of ROS ensures is a straightforward task), then we would maintain the ability to view the designed behaviours operate in the 'real world', thus adding the application dimension to the learners experience - just as we intend to achieve for the children designing dialogue interactivity for the museum guide robot.

\section{Conclusion}

Our intention in this paper was to describe a system we have developed for engaging learners in a wide range of contexts with the design of dialogue-based interactivity for mobile robots, and more specifically in this case, with application to the heritage domain. With an emphasis on a web-based solution that is grounded in both a real robot system and a real application domain, our intent is to enhance the benefits to both driving research through potential usergroup engagement, and enhancing motivation by providing a real application context for the learners involved. In a practical sense, the system is scalable to large numbers of simultaneous learners (as shown in the first case study for example), but also to a wide range of learners (as shown by the very different demographics of the participants of the two case studies). Our observations from preliminary events support the view that the system is both usable and successful in supporting engagement with the problem presented to the participants, with indications that this can persist over an extended period of time. It remains to be seen whether such high engagement will result in tangible learning outcomes/benefits, however, we have put in place a system that facilitates such efforts.

\section{References}

1. Baxter, P., Ashurst, E., Kennedy, J., Senft, E., Lemaignan, S., Belpaeme, T.: The Wider Supportive Role of Social Robots in the Classroom for Teachers. In: 1st Int. Workshop on Educational Robotics at the Int. Conf. Social Robotics. Paris, France (2015) 
2. Cakmak, M., Takayama, L.: Teaching People How to Teach Robots: The Effect of Instructional Materials and Dialog Design. In: HRI. pp. 431-438. ACM/IEEE, Bielefeld, Germany (2014)

3. Chowdhury, G.: Natural Language Processing. Annual Review of Information Science and Technology 37, 51-89 (2003). https://doi.org/10.1002/aris.1440370103

4. Del Duchetto, F., Kucukyilmaz, A., Iocchi, L., Hanheide, M.: Do not make the same mistakes again and again: Learning local recovery policies for navigation from human demonstrations. IEEE Robotics and Automation Letters 3(4), 4084-4091 (2018). https://doi.org/10.1109/LRA.2018.2861080

5. Diprose, J., MacDonald, B., Hosking, J., Plimmer, B.: Designing an API at an appropriate abstraction level for programming social robot applications. Journal of Visual Languages \& Computing (2016). https://doi.org/10.1016/j.jvlc.2016.07.005

6. Fischer, K., Lohse, M.: Shaping naive users' models of robots' situation awareness. In: RO-MAN 2007 - The 16th IEEE International Symposium on Robot and Human Interactive Communication. pp. 534-539 (2007). https://doi.org/10.1109/ROMAN.2007.4415144

7. Hanheide, M., Göbelbecker, M., Horn, G.S., Pronobis, A., Sjöö, K., Aydemir, A., Jensfelt, P., Gretton, C., Dearden, R., Janicek, M., Zender, H., Kruijff, G.J., Hawes, N., Wyatt, J.L.: Robot task planning and explanation in open and uncertain worlds. Artificial Intelligence 247, 119-150 (2017). https://doi.org/10.1016/j.artint.2015.08.008

8. Horn, M.S., Solovey, E.T., Crouser, R.J., Jacob, R.J.: Comparing the use of tangible and graphical programming languages for informal science education. In: Proceedings of the SIGCHI Conference on Human Factors in Computing Systems. pp. 975-984. CHI '09, ACM, New York, NY, USA (2009). https://doi.org/10.1145/1518701.1518851

9. Karreman, D.E., van Dijk, E.M.A.G., Evers, V.: Contextual Analysis of Human Non-verbal Guide Behaviors to Inform the Development of FROG, the Fun Robotic Outdoor Guide. In: Salah, A.A., Ruiz-del Solar, J., Meriçli, Ç., Oudeyer, P.Y. (eds.) Human Behavior Understanding. pp. 113-124. Springer Berlin Heidelberg, Berlin, Heidelberg (2012)

10. Kopp, S., Gesellensetter, L., Krämer, N.C., Wachsmuth, I.: A conversational agent as museum guide-design and evaluation of a real-world application. In: Intelligent virtual agents. pp. 329-343. Springer (2005)

11. Lee, C., Jung, S., Kim, K., Lee, D., Lee, G.G.G.: Recent approaches to dialog management for spoken dialog systems. Journal of Computing Science and Engineering 4(1), 1-22 (2010). https://doi.org/10.5626/JCSE.2010.4.1.001

12. McNerney, T.S.: From turtles to Tangible Programming Bricks: Explorations in physical language design. Personal and Ubiquitous Computing 8(5), 326-337 (2004). https://doi.org/10.1007/s00779-004-0295-6

13. Papert, S.: Mindstorms: Children, Computers, and Powerful Ideas. Basic Books, Inc., New York, NY, USA (1980)

14. Šabanović, S.: Robots in Society, Society in Robots. International Journal of Social Robotics 2(4), 439-450 (2010). https://doi.org/10.1007/s12369-010-0066-7

15. Thrun, S., Bennewitz, M., Burgard, W., Cremers, A.B., Dellaert, F., Fox, D., Hahnel, D., Rosenberg, C., Roy, N., Schulte, J., Schultz, D.: Minerva: A secondgeneration museum tour-guide robot. In: Proceedings of the 1999 IEEE International Conference on Robotics and Automation. IEEE (1999) 